\title{
PAPER
}

\section{Nap-dependent learning in infants}

\section{Almut Hupbach, Rebecca L. Gomez, Richard R. Bootzin and Lynn Nadel}

Department of Psychology, The University of Arizona, USA

\begin{abstract}
Sleep has been shown to aid a variety of learning and memory processes in adults (Stickgold, 2005). Recently, we showed that infants' learning also benefits from subsequent sleep such that infants who nap are able to abstract the general grammatical pattern of a briefly presented artificial language (Gomez, Bootzin \& Nadel, 2006). In the present study, we demonstrate, for the first time, long-term effects of sleep on memory for an artificial language. Fifteen-month-old infants who had napped within 4 hours of language exposure remembered the general grammatical pattern of the language 24 hours later. In contrast, infants who had not napped shortly after being familiarized with the language showed no evidence of remembering anything about the language. Our findings support the view that infants' frequent napping plays an essential role in establishing long-term memory.
\end{abstract}

\section{Introduction}

Sleep plays a beneficial role for a variety of cognitive functions. For instance, nocturnal sleep following learning has been shown to support the consolidation of newly formed memories (Stickgold, 2005), and sleep can facilitate the extraction of explicit knowledge (Fischer, Drosopoulos, Tsen \& Born, 2006) and insight into a problem solution (Wagner, Gais, Haider, Verleger \& Born, 2004). Fewer studies have addressed the effects of daytime naps. Naps containing both slow-wave sleep (SWS) and rapid eye-movement (REM) sleep have been shown to be as beneficial as nocturnal sleep for a visual discrimination task (Mednick, Nakayama \& Stickgold, 2003). Naps containing only SWS do not improve procedural memory performance but positively affect explicit memory (Tucker, Hirota, Wamsley, Lau, Chaklader \& Fishbein, 2006). With regard to development, sleep is assumed to be involved in brain maturation and synaptic plasticity during early life (Frank, Issa, Stryker \& Keck, 2001; Maquet, Stickgold \& Smith, 2003); however, most of the data supporting this hypothesis come from studies with nonhuman animals. Although sleep-wake state organization is a predictor of future cognitive status in infancy (Gertner, Greenbaum, Sadeh, Dolfin, Sirota \& Ben-Nun, 2002), little is known about the direct effects of sleep, especially napping, on infants' learning and memory. We recently showed that a daytime nap facilitates abstraction, an ability that is critical for the developing cognitive system (Gomez et al., 2006). Specifically, 15month-old infants were exposed to an artificial language consisting of three-word strings in which the first word always predicted the third word, and the middle element varied, promoting nonadjacent dependency learning. One group napped during the interval between familiarization and test; another group did not. Infants who had not napped in the interval between familiarization and test preferred to listen to familiar strings (i.e. the exact strings they had listened to during exposure) in comparison to new, unfamiliar strings (i.e. test strings that contained the same elements but violated the previously learned rule), thus showing evidence for veridical memory for specific nonadjacent pairs. In contrast, infants who had napped showed no veridical memory effect; instead, their preference was determined by the very first string they heard during test: if the test started with a familiar test string, they listened longer to familiar than to unfamiliar strings. If the first string was unfamiliar, they listened longer to unfamiliar than to familiar test strings. We interpreted this as showing that sleep facilitates the abstraction of a more general rule about the language, namely the relationship between the first and third word, independent of the specific language infants were exposed to before. This abstraction is triggered by the first nonadjacent dependency infants hear at test.

In Experiment 1 we ask whether the abstraction effect is a transient phenomenon or whether sleep has some long-term effects that can be detected even 24 hours after language exposure. Our previous results (Gomez et al., 2006) could be explained by the fact that infants who had napped between familiarization and test were more rested at the time of test in comparison to infants who had not napped in that interval. Therefore, in Experiment 1, infants were exposed to the artificial 
language and tested a day later at a time of day that fell right before their nap. In Experiment 2 we ask whether this abstraction effect is dependent upon an immediate nap or whether any sleep within a 24-hour period will suffice.

\section{Experiment 1}

To test the long-term effects of sleep on memory for an artificial language, 15-month-old infants were exposed to an artificial language at a time that fell right before their usual daytime nap, such that all infants napped within the following 4 hours, and were then tested 24 hours after language exposure. The children listened to an artificial language requiring them to track sequential dependencies between the first and the third words in three-word sentences (e.g. pel-wadim-jic, vot-kicey-rud, pel-deecha-jic). The middle element in the strings came from a large set of words. This pattern resembles dependencies between auxiliaries and inflections in English (e.g. is playing) where there are a limited number of auxiliaries and inflections, and dependencies between them, but a large number of verb stems. Infants were tested 24 hours after being familiarized with the language, using the head-turn preference procedure (Kemler Nelson, Jusczyk, Mandel, Myers, Turk \& Gerken, 1995) to measure listening times to familiar and unfamiliar strings (e.g. in familiar strings pel predicted jic and vot predicted $r u d$; in unfamiliar strings pel predicted rud and vot predicted jic). Importantly, familiar and unfamiliar strings could be distinguished only by noting the longdistance relationship between the first and third words.

If the abstraction effect in our previous study (Gomez et al., 2006) is a transient phenomenon, we should find no evidence of it 24 hours later. If, however, sleep causes a more stable and long-lasting abstraction of the grammatical pattern, we should still observe this effect 24 hours later.

\section{Method}

\section{Participants}

Twenty-four healthy 15-month-olds (12 girls, 12 boys, average age: 15.23 months), recruited from the Tucson area, participated in the experiments. On the first day of their participation they were exposed to the language at a time of day when they were expected to take a nap within 4 hours. Eight infants were discarded from the initial sample, because parents interfered with the test procedure $(n=2)$, the infants were upset and cried during test $(n=4)$, or because of technical difficulties $(n=2)$, leaving 24 participants.

\section{Materials}

Infants were familiarized with one of two versions of the artificial language. Strings took the form pel-X-jic and
vot-X-rud in Version $\mathrm{A}$, and pel-X-rud and vot-X-jic in Version B. Twenty-four X elements were used producing 48 three-element strings in each version of the language. The $\mathrm{X}$ elements were wadim, kicey, puser, fengle, coomo, loga, gople, taspu, hiftam, deecha, vamey, skiger, benez, gensim, feenam, laeljeen, chila, roosa, plizet, balip, malsig, suleb, nilbo, and wiffle. Half of the infants in each experiment were familiarized with Version A, and half with Version B of the artificial language.

\section{Familiarization stimuli}

A female speaker recorded sample strings. Word tokens from the recorded strings were used to construct Version $\mathrm{A}$ and $\mathrm{B}$ strings (using the same tokens in both versions eliminates differences in the pronunciation of words or strings in the two versions, so that the nonadjacent dependencies are the only source of difference). Strings were separated by 1,000-ms pauses. Infants heard each of the 48 strings of their training language five times during familiarization. Thus, they heard each of the two nonadjacent word dependencies 120 times.

\section{Test stimuli}

Six strings in each version of the language were used as test items. Four test sets were created - the Version A strings and Version B strings - each presented in two orders. Each test set was presented four times (once in each of four test blocks), for a total of 16 trials (i.e. a trial consisted of the presentation of a test set). Test sets were 17 seconds in duration.

\section{Procedure}

On the first day, the experimenter visited the infant's home and played a 15-minute recording of one of the artificial language versions from a tape recorder while interacting quietly with the infant. Additionally, the experimenter attached a Minimitter ${ }^{\circledR}$ Actiwatch with a memory chip to the infant's ankle. The actiwatch records body movements and provides an accurate estimate of the infant's sleep behavior (Acebo, Sadeh, Seifer, Tzischinsky, Hafer \& Carskadon, 2005). Caregivers were also asked to keep a $\log$ of the infant's sleep behavior, noting periods of sleep and wakefulness as well as externally produced motion (e.g. car ride).

On the second day ( 24 hours after familiarization), infants were tested in the head-turn preference procedure in the lab at the University. The infant was seated on the caregiver's lap in an enclosed test booth. A center light, directly in front of the infant, was used to gain his or her attention at the beginning of a trial. Two side lights, mounted directly underneath audio speakers on the infant's left and right, were used to draw the infant's attention to the speaker that would emit the stimulus on a given trial. Side of presentation was determined randomly, with familiar and unfamiliar test trials played from each side. 
An observer outside the booth monitored the infant's looking behavior using a computer program. The computer program initiated test trials in random order and recorded direction and duration of head-turn responses. Each of the 16 test trials began with the center light blinking. Once the infant fixated the center light, the light was extinguished, initiating blinking of a side light. When the infant made a head turn of at least 30 degrees in the direction of the blinking side light, the sample began to play, continuing until its completion or until the infant failed to maintain the minimum 30-degree head turn for 2 consecutive seconds (signaling the infant's loss of attention). The dependent measure was the amount of time the infant oriented toward the test stimulus.

\section{Results}

Time of day for the familiarization and test phases was the same for individual infants but varied between the infants from 8:30 a.m. to 2:00 p.m. with an average test time of 11:13 a.m. $(S D=96 \mathrm{~min})$. Actigraphy data showed that the average time delay between exposure to the language and the onset of the nap was 111 minutes $(S D=61 \mathrm{~min})$, and the average nap duration was 84 minutes $(S D=32 \mathrm{~min})$. The average time delay from exposure to the onset of nocturnal sleep was 547 minutes $(S D=81 \mathrm{~min})$. On average, infants were awake for 169 minutes $(S D=95 \mathrm{~min})$ prior to the test on Day 2 .

Mean looking-time differences between trials consistent with and inconsistent with the first test trial differed significantly from zero, $M=1.10 \mathrm{~s}, \mathrm{SEM}=.39 \mathrm{~s}, t(23)=2.79$, $p=.01$. Replicating the abstraction effect found in Gomez et al. (2006), if the test started with a familiar test string, infants listened longer to familiar than to unfamiliar strings for the remainder of the test. If the first string was unfamiliar, infants listened longer to unfamiliar than to familiar test strings. The data can also be scored according to veridical memory to determine if infants listen longer to familiar strings than to ones they did not hear during familiarization. We found no evidence for such an effect (mean looking-time differences between familiar and unfamiliar test strings did not differ significantly from zero, $M=-.51 \mathrm{~s}, S E M=.44 \mathrm{~s}, t=-1.15, p=.26$ ).

Mean looking-time differences between trials consistent with and inconsistent with the first test trial (the abstraction effect) did not significantly correlate with nap duration $(r=.164)$ or with the time delay between exposure to the language and onset of napping $(r=-.103)$. Mean looking-time differences between familiar and unfamiliar trials (veridical memory) also did not correlate with the nap length $(r=-.159)$ or with time delay between exposure and onset of napping $(r=-.096, t<1$ for all correlations).

\section{Discussion}

Consistent with the abstraction effect found in our first sleep study (Gomez et al., 2006), infants' head-turn preference was determined by the very first trial type they heard during test (regardless of whether strings consisted of familiar or unfamiliar nonadjacent dependencies). This suggests that sleep facilitated the abstraction of a more general rule about the language, one based on the predictive relationship between the first and third word very generally such that the relationship is independent of a specific match in vocabulary between training and test. Our results indicate that the abstraction effect is a stable phenomenon that can be observed in 15-monthold infants not only shortly after the infants wake up from their nap (Gomez et al., 2006), but even after a 24hour delay. Additionally, this effect is not contingent upon infants being well rested at the time of test, because in contrast to our previous study, the present study tested infants right before their regular daytime nap.

The duration of the nap was not correlated with the abstraction effect. However, our criterion for a nap was a priori set to a sleep duration of at least 30 minutes. Therefore, we do not know whether shorter sleep episodes would be sufficient for the abstraction effect to develop. A recent study with adults shows that for explicit memory performance, even an ultra short episode of sleep has a beneficial effect (Lahl, Wispel, Willigens \& Pietrowsky, 2008). The data of our second experiment, in which a few infants napped for less than 30 minutes, suggest that shorter episodes of sleep do not promote abstraction in our paradigm: although the sample size was small, none of the three infants who napped for a short time after language exposure showed differences consistent with an abstraction effect (i.e. looking times for trials consistent with the first trial were not longer than looking times inconsistent with the first trial). Additionally, our sample was fairly small with 24 infants, and infant data are notoriously variable. It may be that significant correlations would be observed with a larger sample.

Importantly, the nap that fell in the 4-hour window after familiarization was not the only time infants slept during the 24-hour period between exposure and test. Some infants took another nap later in the day, and all infants slept during the night. Therefore, it remains an open question whether infants need to sleep shortly after language exposure for the abstraction effect to occur or whether any sleep during the 24-hour period will cause a similar effect. We investigated this question in the next experiment.

\section{Experiment 2}

In a second experiment, we exposed infants to the language at a time of day when they were not expected to take a nap during the following 4 hours, and they were again tested 24 hours later. Thus, in contrast to Experiment 1, these infants did not nap shortly after listening to the language, but all infants slept in the 24-hour interval between exposure and test. 
Method

\section{Participants}

Twenty-four infants (12 girls, 12 boys, average age: 15.30 months) were scheduled at a time of day when they were expected not to take a nap within 4 hours of language exposure. ${ }^{1}$ We set the criterion for 'no-napping' in the 4hour interval after language exposure to a sleep duration of less than 30 minutes. Three infants slept between 15 and 20 minutes in the 4-hour interval after language exposure. Eleven infants were discarded from the initial sample because they slept more than 30 minutes in the 4-hour interval following familiarization $(n=5)$, because parents interfered with the test procedure $(n=1)$, the infants were upset and cried during test $(n=4)$, or because of technical difficulties $(n=1)$.

\section{Materials and procedure}

The materials and procedure were identical to Experiment 1 . The only difference between the two experiments was that in Experiment 1, infants napped in the 4-hour interval after language familiarization, whereas in Experiment 2, infants did not nap in the 4-hour interval after familiarization.

\section{Results}

Time of day for familiarization and test varied between the infants from 8:30 a.m. to 4:30 p.m., with an average test time of 1:49 p.m. $(S D=136 \mathrm{~min})$. Five infants took a nap later in the day, while 19 infants did not take a nap after language exposure; instead, they did not sleep until they were put down for their nighttime sleep (both the nap and the nighttime sleep took place outside of the 4-hour window following language exposure). Actigraphy data showed that the average time delay between exposure to the language and when the infants were put to bed (for a nap or nighttime sleep) was 327 minutes $(S D=70 \mathrm{~min})$, and the average duration for the sleep episode following language exposure was 546 minutes $(S D=236 \mathrm{~min})$. The average time delay from language exposure to the onset of nocturnal sleep was 394 minutes $(S D=154 \mathrm{~min})$. On average, infants were awake for 92 minutes $(S D=80 \mathrm{~min})$ prior to the test on Day 2 .

Mean looking-time differences between trials consistent with and inconsistent with the first test trial did not differ significantly from zero, $M=.28 \mathrm{~s}, S E M=.64 \mathrm{~s}$, $t<1, p=.67$, thus there was no abstraction effect. There was also no evidence for a veridical memory effect (mean looking-time differences between familiar and unfamiliar

\footnotetext{
${ }^{1}$ Infants in the two experiments did not differ with regard to duration or pattern of nighttime sleep, overall sleep duration within the 24 hours between familiarization and test, parents' educational level, season when testing occurred, or language comprehension and production. None of the infants were exposed to a second language on a regular basis.
}

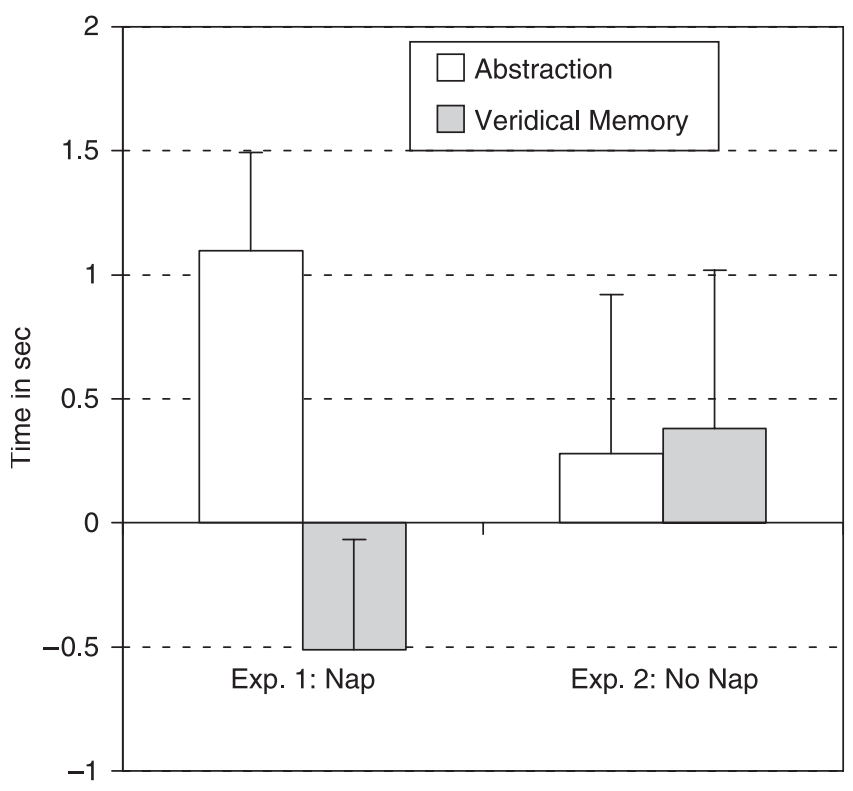

Figure 1 Mean looking-time differences for the abstraction effect (difference between trials consistent and inconsistent with the first test trial) and the veridical memory effect (difference between trials consistent and inconsistent with the training language) in the group that napped (Experiment 1) and in the group that did not nap shortly after language exposure (Experiment 2). All infants were tested with the head-turn preference procedure 24 hours after familiarization with the training language. The error bars represent standard errors of the means.

test strings did not differ significantly from zero, $M=.38 \mathrm{~s}$, $S E M=.64 \mathrm{~s}, t<1, p=.56)$. Looking-time differences for both Experiments 1 and 2 are shown in Figure 1.

\section{Discussion}

Infants who did not nap within an average of 5 hours and 27 minutes after language exposure showed no evidence of abstracting the grammatical regularity nor did they remember the specific language they were exposed to when tested 24 hours later (Experiment 2). Taken together with the results of infants who did nap (Experiment 1), it appears that retaining any information about the language over a 24-hour period requires a nap relatively soon after acquisition.

Infants in Experiments 1 and 2 were scheduled at times that preceded or followed their normal nap times to increase the likelihood that they would take a nap or would not sleep within the 4 hours following language exposure, respectively. This resulted in most infants in Experiment 1 participating in the morning or early afternoon, and most infants in Experiment 2 participating around noon or in the afternoon. Thus, time of test differed between the two experiments. In order to rule out the explanation that results differ due to time of day, we re-analyzed a subset of the data including only infants who participated between 11:00 a.m. and 2:30 p.m., because 
these test times overlapped in the two experiments. As in the analyses of the whole data set, infants who had not napped within an average 5 hours and 27 minutes after familiarization showed no evidence of abstraction $(n=9$, $M=.20 \mathrm{~s}, S E M=1.04 \mathrm{~s}, t<1)$ or veridical memory of the nonadjacent dependencies $(M=-.21 \mathrm{~s}, S E M=1.04 \mathrm{~s}$, $t<1)$. In contrast, infants who napped showed an abstraction effect $(n=11, M=.80 \mathrm{~s}, S E M=.44 \mathrm{~s}, t(10)=1.80$, $\mathrm{p}=.05$, one-tailed), but no veridical memory effect $(M=-.68 \mathrm{~s}, S E M=.46 \mathrm{~s}, t(10)=1.46, p=.09$, one-tailed $)$. This shows that although time of test differed in Experiments 1 and 2, this difference cannot explain the differential findings.

Additionally, most infants in the nap group (Experiment 1) were tested prior to napping on the second day, while most infants in the no-nap group (Experiment 2) were tested after napping. More specifically, infants in the nap group had on average been awake for 169 minutes ( $S D=95 \mathrm{~min}$ ) before testing on Day 2, whereas infants in the no-nap group had been awake for only 92 minutes $(S D=80 \mathrm{~min})$. However, there is no reason to assume that being less tired could hinder the abstraction effect. In our previous study (Gomez et al., 2006), infants in the nap group were the ones who were tested after napping and these infants showed the abstraction effect. Thus, it seems that being well rested has no influence on the abstraction effect.

\section{General discussion}

Our previous work (Gomez et al., 2006) showed that infants who napped in a 4-hour interval between familiarization with an artificial language and test formed an abstraction regarding nonadjacent dependencies in the language whereas infants who did not nap remembered specific nonadjacent dependencies. Infants in the nap group seemed to be primed to detect nonadjacent structure regardless of the actual words instantiating it. The present study suggests that specific nonadjacent dependencies are completely forgotten 24 hours after exposure to the language, whereas abstract information is retained, but only if a nap follows shortly after language exposure. This is an important finding when contrasted with adult research that shows a benefit of nighttime sleep even though participants do not take a nap.

In particular, studies with adults show improved performance on explicit and procedural memory tasks after a good night's sleep (for a review, see Walker \& Stickgold, 2006). In contrast, in our study infants did not improve after a night's sleep if there was a long gap between learning and sleep. One possibility for why sleep must be more immediate is that infants retain information for shorter periods of time than older children and adults (e.g. Richmond \& Nelson, 2007). Specifically, there are dramatic changes during the first 2 years of life in explicit memory and the brain structures that support them. Those changes emphasize the need for more developmental studies on sleep and memory. At the same time, it is not entirely clear which form of memory underlies the effects in our artificial language paradigm, and therefore, a more immediate sleep episode might be important for this form of learning regardless of age. Future studies are needed to address these questions.

Because Gomez et al. (2006) and the present study are the only two studies on the effects of sleep on cognitive performance in infants, we do not know whether the beneficial effect of sleep is task-specific or could be replicated in other paradigms with infants. Future studies need to look at the effects of sleep, and the importance of the timing of sleep after encoding for other memory and language learning paradigms in infants, in order to draw more general conclusions.

The abstraction effect is a rather unusual result in the infant literature, but the fact that we have replicated it in the present study suggests that it is not a spurious phenomenon. Furthermore, as demonstrated in Gomez et al. (2006), this effect does not occur in a control group exposed to a low variability learning condition. ${ }^{2}$ Thus, at a minimum we know that the effect documented here stems from the learning of nonadjacent structure. Although an effect dependent on the first test trial is not predicted in the infant literature, it makes sense within a broader theory of learning. By this view, learning is seen as a process unfolding over time, altered by consolidation and sleep as well as additional experience with the same (or similar) information. What infants retain from brief exposure to novel information (the learning conditions here) will be very different from retention after multiple exposures to the same or similar information. Retention will also be affected by the delay between exposure and test (most learning studies test infants immediately after exposure). On initial exposure, infants could either retain very specific information or they could retain a broad generalization. Our studies (this one and Gomez et al., 2006) suggest that although infants can retain specific information up to 4 hours after familiarization, what they retain over 24 hours is abstract in nature. From a learning point of view such retention is adaptive because while the infant is primed to look for similar structure under similar learning conditions, s/he is not locked into looking for a specific instantiation of this structure (more on this below). Additional exposure to the same structure presumably results in a more specific memory.

In conclusion, it might be especially adaptive for a developing organism to recognize similar but slightly different instances and to apply rules flexibly to incoming information. That is, if memories were specific to an exact form as opposed to an abstract rule characterizing that form, learners would be less likely to recognize new instances that follow the same rule but differ slightly on

\footnotetext{
${ }^{2}$ A critical manipulation in this paradigm is the size of the set from which we draw the middle item. When the set is large $(X=24)$ infants are able to detect the nonadjacent structure. When set size is small, infants do not learn the nonadjacent dependency.
} 
a surface level. In contrast, a learner who is sensitive to the abstract rule can detect that rule despite small changes in surface form. This sensitivity might allow a developing organism to acquire a stable core of rules with flexibly interchangeable elements. In adults, sleep occurring up to 12 hours after a learning experience initiates long-lasting changes in the representation of memories, but sleep occurring 24 or more hours later does not result in a permanent change (Gais, Albouy, Boly, Dang-Vu, Darsaud, Desseilles, Rauchs, Schabus, Sterpenich, Vandewalle, Maquet \& Peigneux, 2007). Our data suggest that the window of time during which sleep has a beneficial impact on memory is much narrower for infants: They need to nap within 4 hours of learning to extract and retain information about a regularity from the incoming stream of stimulation. Thus, infants appear to need frequent periods of sleep or rest to code and consolidate regularities present in the continuous stream of new information they are exposed to every day.

\section{Acknowledgements}

This research was funded by NSF CAREER award BCS-0238584 to RLG. We thank K. Fridel for assistance with actigraphy analysis and the parents and infants who participated in this study.

\section{References}

Acebo, C., Sadeh, A., Seifer, R., Tzischinsky, O., Hafer, A., \& Carskadon, M. (2005). Sleep/wake patterns derived from activity monitoring and maternal report for healthy 1 - to 5-year-old children. Sleep, 28, 1568-1577.

Fischer, S., Drosopoulos, S., Tsen, J., \& Born, J. (2006). Implicit learning - explicit knowing: a role for sleep in memory system interaction. Journal of Cognitive Neuroscience, 18, 311-319.

Frank, M., Issa, N., Stryker, M., \& Keck, W. (2001). Sleep enhances plasticity of the developing visual cortex. Neuron, 30, 275-287.
Gais, S., Albouy, G., Boly, M., Dang-Vu, T.T., Darsaud, A., Desseilles, M., Rauchs, G., Schabus, M., Sterpenich, V., Vandewalle, G., Maquet, P., \& Peigneux, P. (2007). Sleep transforms the cerebral trace of declarative memories. Proceedings of the National Academy of Sciences, USA, 104, 18778-18783.

Gertner, S., Greenbaum, C.W., Sadeh, A., Dolfin, Z., Sirota, L., \& Ben-Nun, Y. (2002). Sleep-wake patterns in preterm infants and 6 month's home environment: implications for early cognitive development. Early Human Development, 68, 93-102.

Gomez, R.L., Bootzin, R.R., \& Nadel, L. (2006). Naps promote abstraction in language-learning infants. Psychological Science, 17, 670-674.

Kemler Nelson, D.G., Jusczyk, P.W., Mandel, D.R., Myers, J., Turk, A., \& Gerken, L. (1995). The head-turn preference procedure for testing auditory perception. Infant Behavior and Development, 18, 111-116.

Lahl, O., Wispel, C., Willigens, B., \& Pietrowsky, R. (2008) An ultra short episode of sleep is sufficient to promote declarative memory performance. Journal of Sleep Research, 17, 3-10.

Maquet, P., Stickgold, R., \& Smith, C. (2003). Sleep and brain plasticity. Oxford: Oxford University Press.

Mednick, S., Nakayama, K., \& Stickgold, R. (2003). Sleepdependent learning: a nap is as good as a night. Nature Neuroscience, 6, 697-698.

Richmond, J., \& Nelson, C.A. (2007). Accounting for change in declarative memory: a cognitive neuroscience perspective. Developmental Review, 27, 349-373.

Stickgold, R. (2005). Sleep-dependent memory consolidation. Nature, 437, 1272-1278.

Tucker, M.A., Hirota, Y., Wamsley, E.J., Lau, H., Chaklader, A., \& Fishbein, W. (2006). A daytime nap containing solely non-REM sleep enhances declarative but not procedural memory. Neurobiology of Learning and Memory, 86, 241247.

Wagner, U., Gais, S., Haider, H., Verleger, R., \& Born, J. (2004). Sleep inspires insight. Nature, 427, 352-355.

Walker, M.P., \& Stickgold, R. (2006). Sleep, memory and plasticity. Annual Review of Psychology, 57, 139-166.

Received: 17 June 2008

Accepted: 15 September 2008 